\title{
Influence of Polyelectrolyte Architecture on the Electro-kinetics and Dewaterability of Industrial MBR Activated Sludge
}

\author{
Shiva A. Yousefi ${ }^{1}$, Mustafa S. Nasser ${ }^{*}$, Ibnelwaleed A. Hussein ${ }^{1}$, Reyad Shawabkeh ${ }^{2}$ \\ ${ }^{1}$ Gas Processing Center, College of Engineering, Qatar University, Doha, Qatar \\ ${ }^{2}$ Department of Chemical Engineering, University of Jordan, Jordan
}

\begin{abstract}
Improvement of sludge dewaterability is greatly hindered by the presence of large amount of water molecules trapped in sludge as a result of its strong hydrophilic characteristics. This study investigates the influence of linear and branched structures of polyacrylamide (PAM) with different charge density (CD) on the electro-kinetics, dewatering and volume reduction of highly stable industrial membrane bioreactor (MBR) sludge. The presence of PAM has induced intensive impact on the flocculation behaviour of the sludge as revealed by supernatant turbidity, zeta potential, capillary suction time (CST), flocs size and settleability. Enhancement of the flocculation and dewatering of sludge is correlated to the surface charge neutralization and bridging mechanisms. The highest optimum dose is obtained as $70 \mathrm{mg} . \mathrm{L}^{-1}$ for linear PAM of $40 \%$ $\mathrm{CD}$ whereas the lowest value is determined as $30 \mathrm{mg} . \mathrm{L}^{-1}$ for branched PAMs. In all cases, turbidity removal of $99.9 \%$ and CST reduction of $51-64 \%$ are attained where the linear PAMs revealed the highest CST reduction. As per the flocs formation, higher PAM dose results in bigger flocs until the saturation point, beyond which the surface saturation occurs. The sludge percent volume reduction is measured and correlated to the flocs size and PAM dose. At an optimum PAM's dose, bigger flocs resulted in higher percent volume reduction, however, at a unified PAM's dose, branched structure of PAM achieved higher percentage volume reduction having smaller flocs size. Overall, the branched structure of polyacrylamide was more efficient in flocculation of MBR sludge at lower dose of $30 \mathrm{mg} . \mathrm{L}-1$ for both 40 and $60 \% \mathrm{CD}$ and attaining the highest percentage volume reduction of $58 \%$.
\end{abstract}

Key words: Activated sludge; Membrane bioreactor; Polyelectrolyte; Polyacrylamide; Charge density; Volume reduction

*Corresponding Author: Dr. Mustafa Nasser, Email: m.nasser@qu.edu.qa, Tel +974 4403-4380, Fax +974 44034371

\section{Introduction}

Membrane bioreactor (MBR) is a recent biological treatment process in many industrial and municipal industries that has been implemented to overcome the current difficulties of activated sludge (AS) processes for separation of biomass from the treated water and the sludge volume reduction. This process produces excess sludge with more than $99 \%$ of water at the bottom of thickeners (Pambou et al., 2016). The produced sludge has a poor dewaterability due to the presence of extracellular polymeric substances (EPS) (Suopajärvi et al., 2017). The large quantities of WAS produced by MBR reactor need to be handled effectively as it causes serious 
environmental problems (Zhou et al., 2017). Waste activated sludge has a complex and unstable nature. It composes of water and dissolved wastewater as a continuous phase and sludge flocs, particulate wastewater, and biological products, organic content and micro-organism are the dispersed phases (Ratkovich et al., 2013). Due to its hydrophilicity nature, it traps a large amount of water molecules within the networked sludge flocs and makes it difficult to be dewatered. Therefore, mechanical dewatering is required to reduce the sludge volume. However, before mechanical dewatering, it is essential to condition the WAS using chemical, physical and/or biological methods to fasten the dewatering step which has a real impact on the subsequent unit operations such as storage, pumping, transportation, and handling, improve sludge quality and reduce the sludge volume and consequently reduce the cost of transportation and ultimate disposal (Novak, 2006).

Coagulation/flocculation process is found to be one of the costs effective, user-friendly and wellknown technology and has been used widely and for a long period of time for pretreatment/conditioning of sludge (Bi et al., 2015; Wei et al., 2018). Reagents used for this purpose comprise chemical conditioners such as organic flocculants (i.e. polyelectrolytes), inorganic coagulants (ferric and aluminum sulfate, lime and iron chloride, etc.) and organic coagulants (polymers with high $\mathrm{CD}$ ) and low MW such as polydiallyldimethylammonium chloride (polyDADMAC) and polyamine (Bolto and Gregory, 2007; Kamaruddin et al., 2017; "SNF Floerger," 2016)

Organic polyelectrolytes such as polyacrylamide (PAM) are generally preferred for sludge conditioning and they are considered as the most efficient flocculating agents among the most important breakthroughs in solid-liquid separations. The flocs formed through charge neutralization and bridging mechanisms using organic polyelectrolyte are sufficiently large and strong and can be separated by physical means like sedimentation, and they are highly resistant to any breakage due to the hydrolysis stress. Therefore, this study aims to investigate the influence of polyelectrolyte-based flocculants (i.e., PAMs) with different molecular architecture (linear and branched) and charge density (CD) on the electro-kinetics, dewatering, and rheological characteristics of highly stable industrial MBR sludge.

\section{Material \& Methods}

\section{Material}

The study was performed on sludge collected fortnightly from the membrane tanks of a small (50 $\mathrm{m}^{3} /$ day capacity) MBR installation at a nearby petroleum industry site in Qatar, the MBR feed comprising a blend of gas processing effluent and gas field produced water. The sampled sludge was immediately transferred to the laboratory and stored at $-4^{\circ} \mathrm{C}$ for no more than four days prior to testing. Table 1 summarizes the characteristics of sludge at the average value of 15-20 measurement for each test.

The four PAM flocculants tested (Table 2), supplied by SNF Floerger (France). 
Table 1: The characteristics of MBR sludge used in this study

\begin{tabular}{l|cccc}
\hline Sludge property & Value & Min & Max & $\begin{array}{c}\text { Standard deviation } \\
\text { (SD) }\end{array}$ \\
\hline TSS, g/l & & & & 0.77 \\
VSS, g/l & 11.2 & 10.1 & 12.5 & 0.67 \\
SVI, ml/g & 10.1 & 9.13 & 11.3 & 3.6 \\
COD, mg/L & 89.9 & 84.3 & 94.9 & 3 \\
[NaCl], mg/L & 82 & 75 & 88 & 3.5 \\
Conductivity, $\boldsymbol{\mu S / c m}$ & 853 & 850 & 857 & 14.7 \\
pH & 1380 & 1362 & 1398 & 0.51 \\
Zeta potential, mV & 7.7 & 7 & 8.3 & 2.25 \\
Turbidity, NTU & -15.37 & -19.53 & -11.18 & 336 \\
D50, um & 2603 & 2048 & 2928 & 2.45 \\
CST, s & 17.12 & 13.42 & 21.77 & 1 \\
\hline
\end{tabular}

Table 2: PAM reagent characteristics

\begin{tabular}{l|ccc}
\hline Name & $\begin{array}{c}\text { Charge } \\
\text { density }(\%)\end{array}$ & $\begin{array}{c}\text { Molecular weight } \\
(\mathbf{g} / \mathbf{m o l})\end{array}$ & Structure \\
\hline FLOPAM FO 4490 SSH & $40 \%$ & $7-10$ million & Linear \\
FLOPAM FO 4690 SSH & $60 \%$ & $7-10$ million & Linear \\
FLOPAM FO 4498 SSH & $40 \%$ & $7-10$ million & Branched \\
FLOPAM FO 4698 SSH & $60 \%$ & $7-10$ million & Branched \\
\hline
\end{tabular}

\section{Methods}

PAM stock solutions of concentration 2 g. $\mathrm{L}^{-1}$ were prepared by stirring the samples in deionized water for 5 hours. Flocculation tests were conducted on the prepared sludge using a jar test apparatus (Stuart Flocculator SW6, UK) comprising six sets of 1L beakers stirred with standard rectangular paddles. Specific volumes (providing the desired of PAM dose to the sludge) of PAM solution were added to $250 \mathrm{ml}$ sludge samples while simultaneously mixing at $180 \mathrm{rpm}$ for $2 \mathrm{~min}$ followed by slow mixing at $50 \mathrm{rpm}$ for $20 \mathrm{~min}$. The resultant flocs were then allowed to settle for $30 \mathrm{~min}$ before extracting the supernatant at a depth of $50 \mathrm{ml}$ below sludge supernatant interface for turbidity (in NTU) and zeta potential $(\zeta, \mathrm{mV}$ ) analysis, using a Hach $2100 \mathrm{~N}$ (US) turbidity meter and Zetasizer ZEN3600 (Malvern Instruments Ltd., UK) respectively. The supernatant was then carefully extracted from the flocculated sludge solids to allow the residual solids volume to be determined before sampling the solids for further analysis of floc size and bulk capillary suction time (CST) using Mastersizer 2000 (Malvern Instruments Ltd., UK) and Triton 319 Multi-purpose CST (Triton Electronics Limited, UK), respectively. Each measurement being replicated 3-5 times 
with a standard deviation (SD) of $0.02-4 \%$. Samples for $\zeta$ determination are pre-filtered to 0.45 $\mu \mathrm{m}$.

\section{Results and Discussion}

\section{Turbidity, Zeta potential and capillary suction time trends}

Figure 1 shows the trends of supernatant turbidity and zeta potential with PAM dose which has been used for the optimum dose selection. There are a variety of $\zeta$ ranges for minimum turbidity removal depending on the treatment processes (Dentel, 1991; Morfesis et al., 2009; Shaikh et al., 2017). In this treatment process, for the water clarity requirement and good quality flocs in terms of type and strength of the surface electrical charges of the sludge particles, the optimum PAM dose was equated to the dose providing the minimum supernatant turbidity of below 2 NTU and $\zeta$ value between \pm 5 for each of the PAM reagents tested (Figure 1). On this basis, the highest optimum doses of 70 and $50 \mathrm{mg} . \mathrm{L}^{-1}$ were associated with $4490 \mathrm{SSH}$ and $4698 \mathrm{SSH}$, linear PAMs with 40 and $60 \% \mathrm{CD}$, respectively, compared with the lowest value of $30 \mathrm{mg} . \mathrm{L}^{-1}$ for $4498 \mathrm{SSH}$ and $4698 \mathrm{SSH}$, branched structured PAMs with 40 and $60 \% \mathrm{CD}$, respectively.
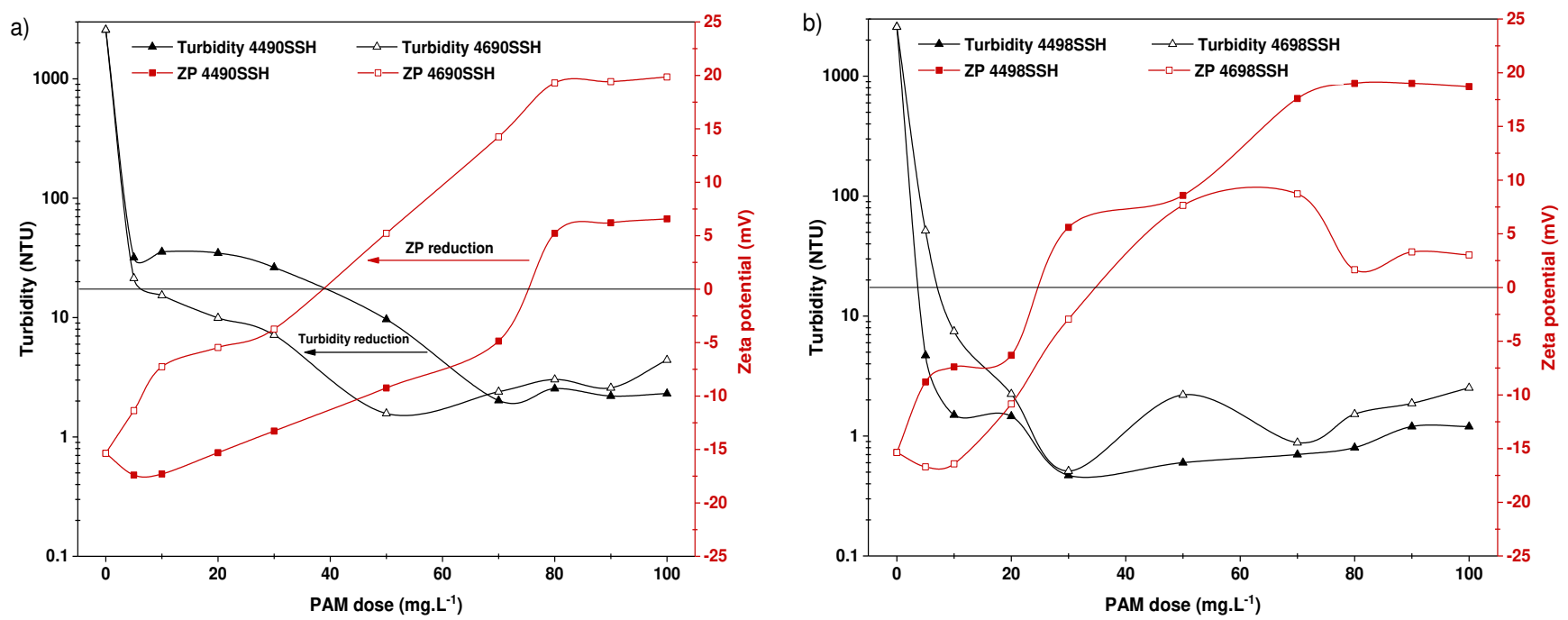

Figure 1: Turbidity $(\mathrm{RSD}=0.02-3 \%)$ and zeta potential $(\mathrm{RSD}=0.02-4 \%)$ of a) linear b) slightly branched c) highly branched

The results indicate all tested polyelectrolytes are being able to achieve charge neutralization at a sufficiently high dose of $30-70 \mathrm{mg} . \mathrm{L}^{-1}$ depending on the polyelectrolyte stricture. The trends evident from the data are:

a) An improved performance (manifested as a decrease in the dose required to achieve a target change in $\zeta$ and reduction in supernatant turbidity) on increasing the CD from $40 \%$ to $60 \%$ for the linear architecture (Figure 1a);

b) Comparability of the attained zeta potential and supernatant turbidity at a given dose between the $40 \%$ and $60 \%$ CD polymers for the branched architectures (Figure 1b).

The supernatant turbidity attained for the branched structured of PAM ; 0.3-0.91 NTU at a dose of $30 \mathrm{mg} . \mathrm{L}^{-1}$, were thus significantly less than the corresponding range of 2-2.5 NTU 
recorded for the linear $4490 \mathrm{SSH}$ polymer at doses above $70 \mathrm{mg} . \mathrm{L}^{-1}$. These trends reflect the efficacy of both $\mathrm{CD}$ and polymer branching on charge neutralization, where branching increases the number of polymer tails with positive ionogenic sites for electrical neutralization of the inherently negatively-charged particles (Nasser and James, 2006). Overall, a 99\% reduction in turbidity was obtained using all types of PAMs.

The results show the significant impact of CD and polymer branching on charge neutralization of the sludge particles. All PAM reagents were able to reverse the negative $\zeta$ values at a specific dose. The required optimum dose depends on PAM type and reflects the preponderance of charge neutralization and polymer bridging. The subsequent reduction in $\zeta$ reduces the repulsive forces between the colloidal particles. Additionally, a bridging mechanism takes place where the long chain of PAM is adsorbed onto the surface of the sludge particles via PAM amide group. The long loops and tails of PAM extend into the solution well beyond the electrical double layer region and attach to the adjacent particles leading to bridging.

CST is used to measure the dewaterability or filterability of the pure and conditioned sludge. High CST value reflects a poor dewaterability, whereas low CST represents a good dewaterability.

CST and turbidity were found to follow similar trends versus PAM dose (Figure 2). The original CST value of $14.8 \pm 1 \mathrm{~s}$ for the unconditioned sludge was reduced to 5.2, 6.6, 7.3 and 7.1 seconds for the FO $4490 \mathrm{SSH}$, FO $4690 \mathrm{SSH}$, FO $4498 \mathrm{SSH}$, and FO $4698 \mathrm{SSH}$, respectively at the optimum PAM doses. CST reduces as the PAM dose increases, however, all tested PAMs resulted in similar CST trends with insignificant difference. An overall reduction of $52-65 \%$ in CST is obtained.

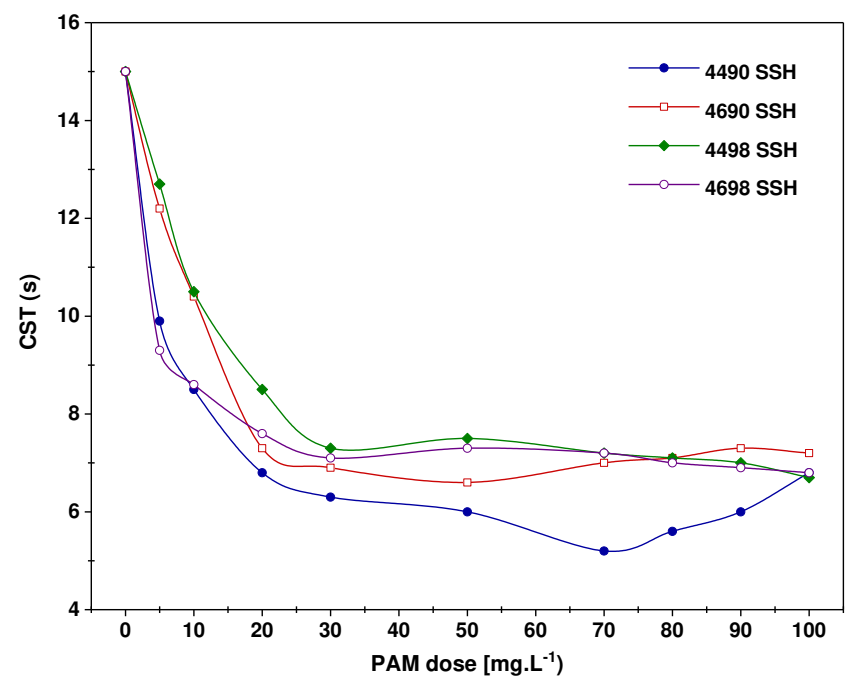

Figure 2. Capillary suction time of $4490 \mathrm{SSH}$ and $4690 \mathrm{SSH}(\mathrm{RSD}=0.5-1 \%)$

\section{Flocs size}

Figure 3 shows the particle size distribution (PSD) of MBR sludge flocculated with different PAMs. Increasing the flocculant dose, increased the surface concentration of PAM on the sludge particle and consequently increased the floc size. For sludge conditioned with FO 4490 SSH PAM, the $D_{50}$ increased from around $17 \mu \mathrm{m}$ (unconditioned sludge) to $260 \mu \mathrm{m}$ at a PAM dose of $70 \mathrm{mg} . \mathrm{L}^{-1}$. Above this dose, surface saturation occurred, and the floc size remained largely unchanged. At the optimum doses of all PAMs, identified on the basis of residual turbidity and $\zeta$, 
the largest flocs (241-246 $\mu \mathrm{m})$ corresponded to the linear PAMs $\left(50\right.$ and $70 \mathrm{mg} . \mathrm{L}^{-1}$ for the 4690 and 4490 SSH products, respectively). Generally, PAMs with higher optimum doses, i.e., FO 4490 SSH and FO 4690 SSH shifted the particle size distribution more toward the right. The lower optimum doses (30 mg. $\mathrm{L}^{-1}$ for the 4498 and $4698 \mathrm{SSH}$ ), yielded much smaller $D_{50}$ values of $155 \mu \mathrm{m}$. This suggests that saturation had not been reached in these cases. However, at a selected unified PAM dose of $70 \mathrm{mg} . \mathrm{L}^{-1}$ (Figure 1b), linear and branched structured of PAM behave similar to each other regardless of PAM characteristics.
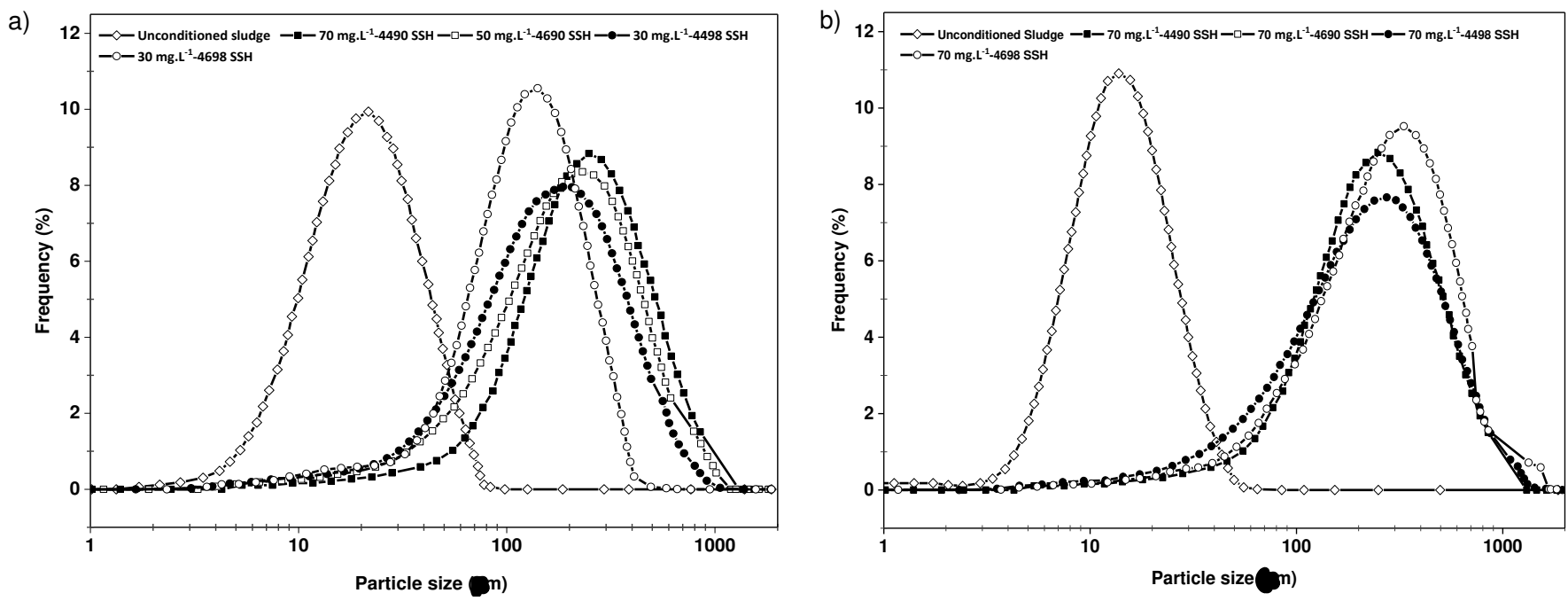

Figure 3: PSD of MBR sludge flocculated at a) optimum dose b) unified dose of all PAMs

\section{Settleability/Volume reduction}

Although a larger floc is preferable in sedimentation processes to improve solid-liquid separation efficiency, this cannot be used alone as a measure of dewaterability or filterability since this does not take into the account floc strength and deformation. Settleability and volume reduction are more appropriate measurements in this regard. Figure 4 shows the percentage volume reduction of MBR sludge that has been measured after extracting the supernatant from the conditioned sludge. According to this figure, at the optimum dose the reduction in volume is directly proportional to PAM's dose and flocs size. However, to assess the effect of CD and structure, a unified dose of $30 \mathrm{mg} . \mathrm{L}^{-1}$ was selected for all PAMs. At this unified dose, the volume reduction increased from 40 to $46 \%$ for a linear PAMs due to the increase in CD from $40 \%$ to $60 \%$. It has been reported that the floc density and strength increase as the PAM's CD increases (Nasser and James, 2008). Additionally, increasing the degree of branching of PAM, resulted in higher percentage volume reduction. 


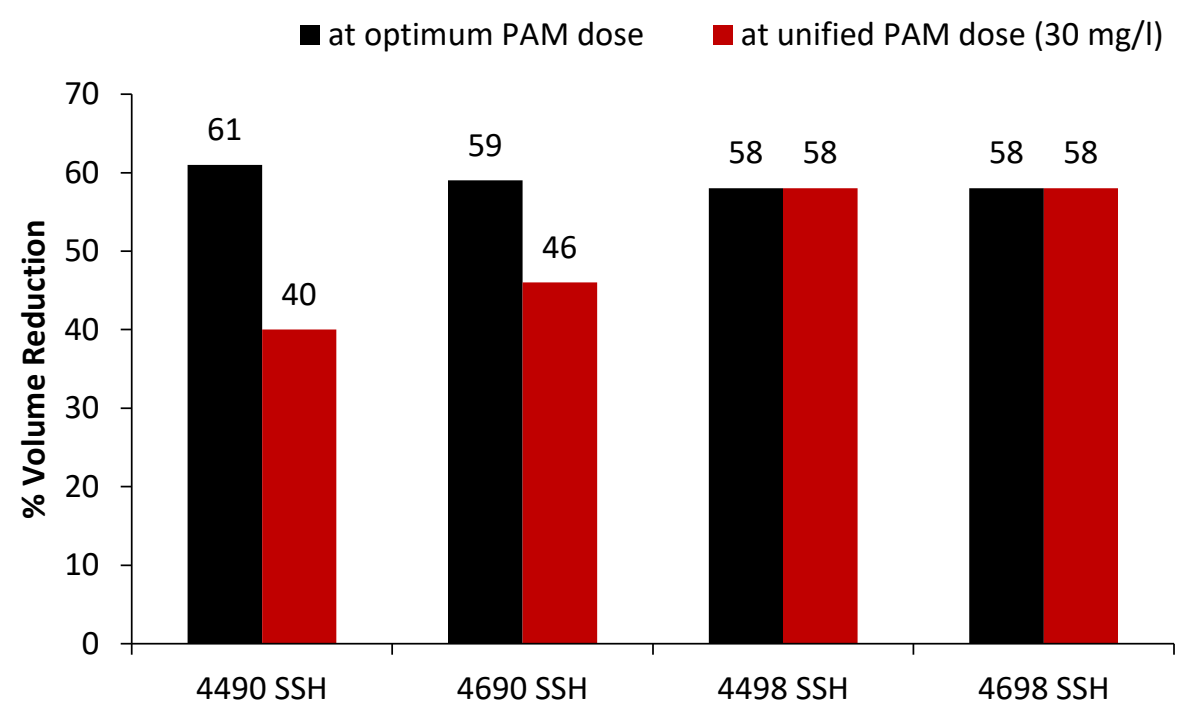

Figure 4. Comparison of \% volume reduction $(\operatorname{RSD}=1-2 \%)$ at optimum and unifiend PAM's dose

\section{Conclusion}

In this study, the effect of polyacrylamide (PAM) structure (linear and branched) and surface charge density on flocculation, namely: charge neutralization, bridging efficiency, volume reduction and dewatering capability of highly stable MBR sludge are examined. This investigation has revealed the following:

$\circ$ PAM structure and charge density distinctly influence the suspension turbidity and zeta potential. Higher $\mathrm{CD}$ is more effective in reducing the particles surface charge and turbidity for linear structure.

- Increasing the degree of branching of PAM exhibits significant influence on lowering supernatant turbidity and zeta potential. So, branched structured of PAMs can be used at low PAM's dose.

- In general, floc size is directly proportional to PAM dose. However, at a unified dose, when branched structure is used, similar values of $D_{50}$ were obtained for all PAMs. Overall, the branched structured PAMs is behaving almost similar to linear PAMs.

- \% volume reduction is directly proportional to PAM's dose and structure such that higher PAM's dose resulted in higher \%volume reduction. However, at a unified dose, branched structured has the highest volume reduction.

\section{Acknowledgments}

The authors would like to acknowledge the support provided by Qatar University. 


\section{References}

Bi, D., Guo, X., Cai, Z., Yu, Z., Wang, D., Wang, Y., 2015. Enhanced dewaterability of wasteactivated sludge by combined cationic polyacrylamide and magnetic field pretreatment. Environ. Technol. 36, 455-462.

Bolto, B., Gregory, J., 2007. Organic polyelectrolytes in water treatment. Water Res. 41, 23012324.

Dentel, S.K., 1991. Coagulant control in water treatment. Crit. Rev. Environ. Control 21, 41-135.

Kamaruddin, M.A., Abdullah, M.M.A., Yusoff, M.S., Alrozi, R., Neculai, O., 2017. CoagulationFlocculation Process in Landfill Leachate Treatment: Focus on Coagulants and Coagulants Aid. \{IOP\} Conf. Ser. Mater. Sci. Eng. 209, 12083.

Morfesis, A., Jacobson, A.M., Frollini, R., Helgeson, M., Billica, J., Gertig, K.R., 2009. Role of Zeta ( ) Potential in the Optimization of Water Treatment Facility Operations 2305-2308.

Nasser, M.S., James, A.E., 2008. Compressive and shear properties of flocculated kaolinitepolyacrylamide suspensions. Colloids Surfaces A Physicochem. Eng. Asp. 317, 211-221.

Nasser, M.S., James, A.E., 2006. The effect of polyacrylamide charge density and molecular weight on the flocculation and sedimentation behaviour of kaolinite suspensions. Sep. Purif. Technol. 52.

Novak, J.T., 2006. Dewatering of Sewage Sludge. Dry. Technol. 24, 1257-1262.

Pambou, Y.B., Fraikin, L., Salmon, T., Crine, M., Léonard, A., 2016. Enhanced sludge dewatering and drying comparison of two linear polyelectrolytes co-conditioning with polyaluminum chloride. Desalin. Water Treat. 57, 27989-28006.

Ratkovich, N., Horn, W., Helmus, F.P., Rosenberger, S., Naessens, W., Nopens, I., Bentzen, T.R., 2013. Activated sludge rheology: A critical review on data collection and modelling. Water Res. 47, 463-482.

Shaikh, S.M.R., Nasser, M.S., Hussein, I.A., Benamor, A., 2017. Investigation of the effect of polyelectrolyte structure and type on the electrokinetics and flocculation behavior of bentonite dispersions. Chem. Eng. J. 311, 265-276.

SNF Floerger [WWW Document], 2016. URL https://www.snf.co.uk/chemical-coagulants-usedwater-treatment/

Suopajärvi, T., Sirviö, J.A., Liimatainen, H., 2017. Cationic nanocelluloses in dewatering of municipal activated sludge. J. Environ. Chem. Eng. 5, 86-92.

Wang, H.-F., Hu, H., Wang, H.-J., Zeng, R.J., 2018. Impact of dosing order of the coagulant and flocculant on sludge dewatering performance during the conditioning process. Sci. Total Environ. 643, 1065-1073.

Wei, H., Gao, B., Ren, J., Li, A., Yang, H., 2018. Coagulation/flocculation in dewatering of sludge: A review. Water Res. 143, 608-631.

Zhou, Y., Zheng, H., Gao, B., Gu, Y., Li, X., Liu, B., Jiménez, A.M., 2017. Waste activated sludge (WAS) dewatering properties of an original hydrophobically modified polyacrylamide containing a cationic microblock structure. RSC Adv. 7, 28733-28745. 